\title{
Fatores relacionados ao diabetes mellitus que podem influenciar no autocuidado
}

RESUMO | Objetivos: Identificar o perfil dos indivíduos maiores de 18 anos, portadores de Diabetes Meliitus (DM) tipo 1 e 2 , de ambos os sexos acompanhados nas ESF da zona rural do município de Divinópolis-MG e os tipos de autocuidado realizados. Método: Estudo descritivo, quantitativo através de um instrumento adaptado e do Questionário de Atividades de Autocuidado com o Diabetes. Resultados: Dentre os 107 entrevistados, 42,1\% têm idade acima de 65 anos, $62,6 \%$ são do sexo feminino com igual porcentagem para os indivíduos que possuem ensino fundamental incompleto e são casados, $65,4 \%$ apresentam outro problema de saúde, sendo $58,7 \%$ Hipertensão Arterial. Na identificação do autocuidado, obteve-se maior prevalência as respostas referentes à "medicação" (96,3\%), ao contrário da "atividade física" (18,7\%), que obteve menor prevalência. Conclusão: a pesquisa evidencia que as pessoas aderem mais ao tratamento medicamentoso do que a mudanças no hábito de vida.

Palavras-chaves: Diabetes Mellitus; Autocuidado; Qualidade de vida.

ABSTRACT | Objectives: To identify the profile of individuals older than 18 years of age, with type 1 and type 2 Diabetes Meliitus (DM), of both sexes followed in the FHS of the rural area of the city of Divinópolis-MG and the types of self-care performed. Method: Descriptive, quantitative study using an adapted instrument and the Diabetes Self-Care Activities Questionnaire. Results: Among the 107 interviewees, $42.1 \%$ are over 65 years of age, $62.6 \%$ are female, with the same percentage for individuals who have incomplete elementary education and are married, $65.4 \%$ have another health problem, being $58.7 \%$ Hypertension. In the identification of self-care, responses to "medication" (96.3\%) were more prevalent, as opposed to "physical activity" $(18.7 \%)$, which had a lower prevalence. Conclusion: the research shows that people adhere more to medication treatment than changes in lifestyle.

Keywords: Diabetes Mellitus; Self-care; Quality of life.

RESUMEN | Objetivos: Identificar el perfil de los individuos mayores de 18 años, portadores de Diabetes Meliitus (DM) tipo 1 y 2 , de ambos sexos acompañados en las ESF de la zona rural del municipio de Divinópolis-MG y los tipos de autocuidado realizados. Método: Estudio descriptivo, cuantitativo a través de un instrumento adaptado y del Cuestionario de Actividades de Autocuidado con la Diabetes. Resultados: Entre los 107 entrevistados, el 42,1\% tiene edad por encima de 65 años, el 62,6\% son del sexo femenino con igual porcentaje para los individuos que poseen enseñanza fundamental incompleta y están casados, el $65,4 \%$ presenta otro problema de salud siendo 58,7\% Hipertensión Arterial. En la identificación del autocuidado, se obtuvo mayor prevalencia las respuestas referentes a la "medicación" (96,3\%), al contrario de la "actividad física" (18,7\%), que obtuvo menor prevalencia. Conclusión: la investigación evidencia que las personas se adhieren más al tratamiento medicamentoso que a los cambios en el hábito de vida.

Palavras claves: Diabetes Mellitus; Autocuidado; Calidad de vida.

\section{Nágila Maria Salomão Souza}

Enfermeira. Graduada em Enfermagem, Universidade do Estado de Minas Gerais, Minas Gerais, Brasil.

ORCID: 0000-0003-1068-3792

\section{Amanda Cristina Cunha}

Enfermeira. Graduada em Enfermagem, Universidade do Estado de Minas Gerais, Minas Gerais, Brasil.

ORCID: 0000-0001-5062-3802

\section{Fernanda Marcelino de Rezende e Silva}

Mestre em Enfermagem pela UFMG, Docente do Curso de Graduação em Enfermagem da Universidade do Estado de Minas Gerais (UEMG), Unidade Divinópolis. ORCID: 0000-0003-2236-7009

Recebido em: 19/07/2020

Aprovado em: 27/07/2020

\section{Karla Amaral Nogueira Quadros}

Enfermeira. Mestre em Educação, Cultura e Organização Social pela Universidade do Estado de Minas Gerais. Minas Gerais, Brasil. Docente do Curso de Graduação em Enfermagem da Universidade do Estado de Minas Gerais (UEMG), Unidade Divinópolis. ORCID: 0000-0002-3750-4873

\section{Regina Consolação dos Santos}

Mestre em Ciências pela Universidade Federal de São João Del Rei, (UFSJ). Docente do Curso de Graduação em Enfermagem da Universidade do Estado de Minas Gerais (UEMG), Unidade Divinópolis.

ORCID: 0000-0002-7393-3210

\section{Silmara Nunes Andradedos Santos}

Enfermeira. Doutora em Ciências da Saúde pela Universidade Federal de São João Del Rei (UFSJ). Docente do Curso de Graduação em Enfermagem da Universidade do Estado de Minas Gerais (UEMG), Unidade Divinópolis.

ORCID: 0000-0002-1975-0827
INTRODUÇÃO

s Doenças Crônicas Não
Transmissíveis (DCNT) têm
aumentado cada vez mais em todo o mundo e têm sido prioridade em saúde pública, alterando de forma intensa a vida da maioria das pessoas. Estas doenças estão relacionadas a riscos ambientais e comportamentais, como o cigarro, a inatividade física, a alimentação inadequada e a obesidade, podendo provocar sentimentos de desespero e aflição, o que implica na necessidade de um cuidado integral a saúde, objetivando uma qualidade e autonomia de suas vidas ${ }^{(1)}$. Destas DCNT, destaca-se o Diabetes Mellitus (DM) por ter alta prevalência em toda a população. Segundo o International Diabetes Federation (IDF), em 2017, estimou-se que a população mundial com DM chegava a cerca de 425 milhões de diabéticos e que 
em 2045 poderá alcançar cerca de 629 milhões de pessoas ${ }^{(2)}$.

Segundo o Ministério da Saúde, o DM é uma doença caracterizada por hiperglicemia e está associada à disfunções, complicações e insuficiência de vários órgãos, dentre eles: rins, olhos, coração, nervos, cérebro e vasos sanguíneos. Pode-se resultar da deficiência da secreção de insulina ou de sua ação, envolvendo processos patogênicos específicos, como destruição das células beta do pâncreas, produtoras de insulina, distúrbios da secreção e resistência à ação da insulina(3).

Com o crescimento populacional, a industrialização, o aumento da expectativa de vida, o maior consumo de dietas hipercalóricas, inatividade física, obesidade e mudanças nos estilos de vida, a prevalência de pessoas com diabetes cresce cada vez mais, ocasionando maior impacto social e econômico, tanto em termos de produtividade, quanto de custos, nesse sentido acometendo principalmente os serviços de saúde. Também interfere diretamente na vida dos pacientes e seus familiares, por trazer consequências como dor, ansiedade e implicações na qualidade e no estilo de vida, além de reduzir a expectativa de vida ${ }^{(4)}$.

Levando em consideração as complicações que o diabetes pode trazer para o portador, reconhecemos que um dos principais componentes do tratamento é o autocuidado, que envolve um plano alimentar, monitoração da glicemia capilar, uso correto da medicação, realização de atividades físicas e o cuidado com os pés, para benefício na manutenção da vida, saúde e bem estar. Destaca-se que essas práticas estão ligadas diretamente a valores, habilidades e limitações ${ }^{(5)}$.

A adoção de medidas de autocuidado diário em pacientes com diabetes é imprescindível para o controle da doença, visto que intervenções no estilo de vida podem influenciar na qualidade de vida, além de prevenir comorbidades. Faz-se necessário que fatores como idade, apoio familiar, escolaridade, acesso à informação, orientação sociocultural, estado de saúde, recursos disponíveis, competências ou déficits de autocuidado, além das características pessoais, sejam identificados para implementação de intervenções mais eficazes, além de ampliar a adesão à terapêutica ${ }^{(6)}$.

Fatores associados ao acréscimo do diabetes mellitus podem ser classificados em: hereditários, socioeconômicos e comportamentais. Evidências científi$\mathrm{cas}^{(7)}$ destacam fatores de risco comportamentais tanto para o aumento de novos casos de DM quanto para elevar o risco de complicações em portadores da doença, tais como: tabagismo; alimentação inadequada com ingestão elevada de alimentos fonte de gorduras, sal e açúcar; sobrepeso e obesidade; sedentarismo; inatividade física; e consumo abusivo de bebidas alcoólicas.

Segundo Oliveira ${ }^{(8)}$, a atuação do enfermeiro é de grande importância para a promoção do autocuidado e no controle do DM, principalmente através de ações educativas voltadas para a promoção da saúde, bem-estar e melhora na qualidade de vida. A educação em saúde e a criação de programas de apoio são estratégias para a implementação de intervenções de enfermagem, e podem ser usadas através de um acompanhamento ao paciente, com mais tempo e número maior de encontros do que os atendimentos de rotina, pois as complicações do DM estão ligadas diretamente ao cuidado pessoal diário adequado e ao estilo de vida saudável.

Logo, tornou-se necessário desenvolver um estudo que avalie quais são os fatores que dificultam o autocuidado dos pacientes diabéticos, que podem influenciar na sua qualidade de vida. Para tanto, o estudo aqui discutido teve por objetivo identificar o perfil dos indivíduos com Diabetes Mellitus acompanhados pelas Estratégias de Saúde da Família da zona rural do município de Divinópolis - MG, segundo variáveis sócio-demográficas, clínicas e relacionadas aos hábitos de vida, diagnóstico e tratamento. Além de identificar quais os tipos de autocuidado são realizados pelos mesmos. 


\section{MÉTODO}

Esse estudo caracteriza-se por ser um estudo descritivo, de abordagem quantitativa, desenvolvido nas Estratégias de Saúde da Família, instituições públicas integradas ao Sistema Único de Saúde de atenção primária, que atendem somente a zona rural do município de Divinópolis-MG. O município está localizado na região centro- oeste de Minas Gerais e possui cerca de 238.230 habitantes $^{(9)}$, sendo 4.362 diabéticos cadastrados ${ }^{(10)}$.

O estudo foi realizado através de entrevista com o paciente e dados coletados do prontuário. A população de estudo foi composta por pessoas maiores de 18 anos, atendidas nas três ESFs de zona rural - Buritis, Quilombo e D'Jalma Dultra - que possuíam diagnóstico de DM tipo 1 e 2, que eram cadastradas no Sistema de Informação de Saúde (SIS) de Divinópolis-MG e tinham classificação de risco de acordo com o Hiperdia. O total de pessoas atendidas pelas ESFs Buritis, Quilombo e D'Jalma Dultra era de 147, de acordo com dados do Secretária Municipal de Saúde de Divinópolis. Para determinação da amostra foi utilizado o cálculo amostral com os critérios estatísticos definidos por Barbetta ${ }^{(11)}$ com erro amostral de $5 \%$.

A entrevista buscou identificar o perfil dos pacientes diabéticos tipo 1 e 2 acompanhados pela ESFs da zona rural do municio de Divinópolis para caracterizar os pacientes com diabetes mellitus segundo variáveis sociodemográficas, clínicas, relacionadas aos hábitos de vida, o diagnóstico e o tratamento.

Os dados foram coletados pelas pesquisadoras no período de setembro a outubro de 2017. Inicialmente os dados seriam coletados após avaliação convencional do enfermeiro ou do profissional médico, no entanto, uma vez que o número de pessoas que viria até a Unidade não seria satisfatório para compor amostra no tempo da coleta já que as ESFs da zona rural cada dia estão em uma comunidade, optou-se por visitar as residências, acompanhados ou não por agentes comunitários de Saúde. A escolha dos participantes foi aleatória dentre os que eram cadastrados no território da ESF e que atendiam os critérios de inclusão da pesquisa. Além disso, foram coletados dados dos prontuários dos participantes.

Para a coleta de dados, primeiramente foi utilizado um instrumento elaborado pelas pesquisadoras, que se baseou em estudos realizados na área e também em literaturas disponíveis ${ }^{(8,12)}$. O mesmo foi estruturado com 30 itens contendo variáveis sociodemográficas, clínicas, e também relacionadas aos hábitos de vida, tratamento e diagnóstico. Em seguida, foi utilizado o Questionário de Atividades de Autocuidado com o Diabetes (QAD), que foi traduzido e adaptado para o Brasil, para verificar com que frequência os entrevistados realizam as atividades ou comportamentos de autocuidado nos setes dias anteriores. Tal possui seis dimensões e quinze itens de avaliação do autocuidado com o diabetes, como alimentação geral, alimentação específica, atividade física, monitorização da glicemia, cuidado com os pés, uso da medicamentação e itens para a avaliação do tabagismo. As
Tabela 1 - Características sociodemográficas dos pacientes diabéticos atendidos pelas Estratégias de Saúde da Familia da zona rural. Divinópolis - MG, 2017.

\section{Variáveis}

Sexo

Feminino

Masculino

Idade (anos)

Até 35 anos
(N)

(\%)

$67 \quad 62,6$

40

37,4 8 respostas variam de 0 a 7 com os escores indicando o autocuidado ${ }^{(13)}$.

Os dados relacionados ao tempo de diagnóstico, terapêutica medicamentosa, peso, altura, valor e data da última glicemia capilar realizada em jejum foram coletados nos prontuários, com exceção daqueles que realizam o teste de glicemia em casa. Os demais dados foram coletados junto ao paciente. Para a aferição da pressão arterial utilizou a técnica padronizada, segundo recomendações da European Dociety of Hypertension ${ }^{(14)}$, o cálculo e a classificação do Índice de Massa Corporal (IMC) foi utilizada a padronização da Organização Mundial de Saúde.

Os dados foram categorizados, transcritos e organizados no programa Excel for Windows, processados e analisados no programa Epi Info 3.5.1. Em seguida, foi analisada a distribuição das variáveis de interesse. Para a apresentação dos mesmos, utilizou-se a estatística descritiva, média, frequência absoluta e porcentagem.

A realização da pesquisa ocorreu após a apreciação e aprovação do Comitê de Ética em Pesquisa em Seres Humanos (CEP) da Universidade Estadual de Minas Gerais (UEMG), mediante o Parecer 2.249.597. A presente pesquisa obedeceu aos preceitos éticos preconizados nas regulamentações descritas na Resolução no 466, de 12 de dezembro de 2012, do Conselho Nacional de Saúde (CNS). O consentimento informado por escrito foi obtido de todos os pacientes.

\section{RESULTADOS}

No grupo estudado (107 pacientes com DM tipo 1 e 2), a maioria (45) tem idade acima de 65 anos (42,1\%). Identificou-se uma predominância (67 pacientes) do sexo feminino $(62,6 \%)$, com igual porcentagem para os indivíduos casados. Quanto à atividade profissional exercida, 32 pacientes $(29,9 \%)$ são do Lar, sendo que 66 pacientes $(61,7 \%)$ moram com de 2 a 4 pessoas na mesma residência e 76 sujeitos possuem renda familiar de até 3 salários mínimos $(71,0 \%)$, com igual porcentagem para as 


\begin{tabular}{|c|c|c|}
\hline De 36-45 anos & 6 & 5,6 \\
\hline De 46-55 anos & 15 & 14,0 \\
\hline De 56 até 65 anos & 33 & 30,8 \\
\hline Acima de 65 anos & 45 & 42,1 \\
\hline \multicolumn{3}{|l|}{ Estado civil } \\
\hline Casado & 67 & 62,6 \\
\hline Solteiro & 16 & 15,0 \\
\hline Divorciado & 6 & 5,6 \\
\hline Viúvo & 18 & 16,8 \\
\hline \multicolumn{3}{|l|}{ Escolaridade } \\
\hline Ens. Fundamental incompleto & 76 & 71,0 \\
\hline Ens. Fundamental completo & 17 & 15,9 \\
\hline Ens. Médio incompleto & 9 & 8,4 \\
\hline Ens. Médio completo & 5 & 4,7 \\
\hline \multicolumn{3}{|l|}{ Renda Familiar* } \\
\hline Entre 0-3 salários mínimos & 76 & 71,0 \\
\hline Entre 3-5 salários mínimos & 23 & 21,5 \\
\hline Acima de 5 salários mínimos & 8 & 7,5 \\
\hline \multicolumn{3}{|c|}{ Pessoas residentes na mesma casa } \\
\hline Mora sozinho (a) & 14 & 13,1 \\
\hline De 2 a 4 pessoas & 66 & 61,7 \\
\hline De 5 a 7 pessoas & 25 & 23,4 \\
\hline Acima de 7 pessoas & 2 & 1,9 \\
\hline \multicolumn{3}{|l|}{ Atividade profissional exercida } \\
\hline Autônomo & 24 & 22,4 \\
\hline Do lar & 32 & 29,9 \\
\hline Trabalhador assalariado & 12 & 11,2 \\
\hline Trabalhador rural & 13 & 12,1 \\
\hline Não exerce atividade profissional & 25 & 23,4 \\
\hline Outras & 1 & 0,9 \\
\hline
\end{tabular}

* Salário mínimo mensal correspondente ao ano de 2017: R\$ 937,00. Fonte: Dados da pesquisa.

Tabela 2 - Dados clínicos dos pacientes diabéticos acompanhados pelas Estratégias de Saúde da Família da zona rural. Divinópolis - MG, 2017.

\begin{tabular}{lcc|} 
Variáveis & (N) & (\%) \\
Índice de massa corporal $(\mathrm{Kg} / \mathrm{m} 2)$ & & \\
\hline Baixo $(<18,5)$ & 2 & 1,9 \\
\hline Normal $(18,5-24,9)$ & 17 & 15,9 \\
\hline Sobrepeso $(25-29,9)$ & 46 & 43,0 \\
\hline Obesidade 1 $(30-34,9)$ & 20 & 18,7 \\
\hline Obesidade 2 (35-39,9) & 18 & 16,8 \\
\hline Obesidade 3 (acima de 40) & 4 & 3,7
\end{tabular}

que possuem ensino fundamental incompleto, como mostra a Tabela 1.

Em relação aos parâmetros antropométricos, utilizou-se especialmente o IMC1. A amostra teve maior prevalência das pessoas que apresentaram sobrepeso $(43,0 \%)$, sendo que nas medidas de circunferência abdominal tanto (43) mulheres $(40,2 \%)$ quanto 18 (homens) $(16,8 \%)$ apresentaram risco muito aumentado (>88/>102), o que demonstra a Tabela 2.

$\mathrm{Na}$ avaliação da pressão arterial, $38,3 \%$ dos diabéticos apresentaram $\mathrm{Hi}$ pertensão Arterial Sistêmica (HAS) tipo 1 (140-159/90-99), dado que vai ao encontro da maior prevalência $(58,7 \%)$ da HAS quando questionados se possuíam outro problema de saúde.

Quanto aos hábitos de vida, verificou-se prevalência entre aqueles (86) que não são tabagistas $(80,4 \%)$, (94) não fazem uso de bebidas alcoólicas (87,9\%), assim como aqueles (88) que não praticavam nenhuma atividade de lazer $(82,2 \%)$. E ao serem questionados sobre o tratamento, apenas $25 \%$ utilizavam insulina, sendo que $20 \%$ utilizavam a NPH humana. (Tabela 3).

$\mathrm{Na}$ avaliação das atividades de autocuidado, obteve-se maior prevalência às respostas referentes à "medicação" $(96,3 \%)$, ao contrário da "atividade física" $(18,7 \%)$, que obteve menor prevalência. Ao analisar a alimentação geral e a específica, nota-se que os participantes realizavam comportamento de autocuidado desejável aos itens propostos, de menos no que concerne ao consumo de alimentos ricos em gorduras. Em relação ao seguimento de uma dieta saudável os indivíduos relataram seguir em média $4,74 \pm 2,25$ dias na semana, a maioria seguia uma dieta de acordo com orientações feitas por profissionais em média $4,33 \pm 2,32$ dias na semana e comiam 5 ou mais porções de frutas e/ou vegetais em média 4,54 2,03 dias na semana. Quanto à restrição de açúcares, os participantes parecem estar conscientes, pois no item consumo de doces obteve-se média de 1,28 $\pm 1,94$ dias na semana. 
Circunferência abdominal (cm)

\begin{tabular}{|c|c|c|}
\hline \multicolumn{3}{|l|}{ Mulher } \\
\hline Normal (Até 80 cm) & 15 & 14,0 \\
\hline Risco aumentado (80-88 cm) & 9 & 8,4 \\
\hline Risco muito aumentado (>88 cm) & 43 & 40,2 \\
\hline \multicolumn{3}{|l|}{ Homem } \\
\hline Normal (Até 90 cm) & 11 & 10,3 \\
\hline Risco aumentado (90-102 cm) & 11 & 10,3 \\
\hline Risco muito aumentado ( >102 cm) & 18 & 16,8 \\
\hline \multicolumn{3}{|l|}{ Pressão arterial (mmHg)2 } \\
\hline Normal $(\leq 120 / \leq 80)$ & 35 & 32,7 \\
\hline Pré-hipertensão (121-139/81-89) & 12 & 11,2 \\
\hline Hipertensão estágio 1(140-159/90-99) & 41 & 38,3 \\
\hline Hipertensão estágio 2 (160-179/100-109) & 12 & 11,2 \\
\hline Hipertensão estágio $3(\geq 180 / \geq 110)$ & 7 & 6,5 \\
\hline \multicolumn{3}{|l|}{ Valor da última glicemia em jejum (mg/dl) } \\
\hline Normal $(<110)$ & 28 & 26,2 \\
\hline Alterada ( $\geq 110)$ & 73 & 68,2 \\
\hline Sem informações & 6 & 5,6 \\
\hline \multicolumn{3}{|l|}{ Data da última glicemia em jejum } \\
\hline Menos de 1 mês & 44 & 41,1 \\
\hline Entre 1-4 meses & 43 & 40,2 \\
\hline Entre 4-6 meses & 7 & 6,5 \\
\hline Acima de 6 meses & 5 & 4,6 \\
\hline Sem informações & 8 & 7,5 \\
\hline
\end{tabular}

Tabela 3 - Caracterização da amostra estudada, segundo dados referentes ao diagnóstico e o tratamento. Divinópolis - MG, 2017.

Variáveis

(N)

Tempo de diagnóstico

\begin{tabular}{lcc} 
De 1 a 10 anos & 82 & 76,6 \\
\hline De 11 a 20 anos & 22 & 20,5 \\
\hline Acima de 20 anos & 3 & 2,8 \\
\hline Tratamento ${ }^{+}$ & & \\
\hline Dieta & 50 & 46,8 \\
\hline Exercício físico & 17 & 15,8 \\
\hline Uso de antidiabético oral & 102 & 95,3 \\
\hline Uso de insulina & 25 & 23,4 \\
\hline Tipo de insulina & & \\
\hline NPH humana & 20 & 18,8 \\
\hline Regular & 3 & 2,7
\end{tabular}

Quanto a prevalência de indivíduos da pesquisa que apresentam sobrepeso, estes seguem uma dieta saudável consumindo frutas e/ou vegetais, mas consomem em média 4,16 $\pm 2,05$ dias na semana, alimentos ricos em gorduras, o que não é favorável para uma alimentação adequada.

No que se refere a prática de atividade física, verifica-se a realização de comportamento não desejável de autocuidado. Pois, no que se refere à realização de qualquer atividade pelo menos 30 minutos ao dia, os indivíduos da pesquisa relataram realizar somente em média $1,91 \pm 2,41$ dias na semana, e no item exercício físico específico foi relatado em média 1,69 2,44 dias na semana, o que mostra que os mesmos não reconhecem este recurso terapêutico como uma prática de autocuidado.

Considerando o tabagismo como um dos hábitos que se relacionam com complicações no tratamento do DM, bem como um dos fatores de risco para o $\operatorname{DM}(7)$, ao serem questionados quanto ao seu histórico em relação ao consumo de cigarros, este estudo mostrou que a prevalência foi daqueles que nunca fumaram $(80,4 \%)$, o que demonstra a realização do autocuidado (Tabela 6).

\section{DISCUSSÃO}

Observando os dados apresentados anteriormente, em relação à faixa etária destacam-se os idosos que, segundo a Organização Mundial de Saúde, são considerados os com idade igual ou superior a 60 anos. Corrobora-se assim para a noção de que o Brasil está passando por mudanças no perfil demográfico e na estrutura etária da população, uma vez que a expectativa de vida tem aumentado, ocasionando um acentuado envelhecimento da população. Tal liga-se diretamente ao crescimento da prevalência e do impacto social das doenças crônicas, principalmente em pessoas com mais de $50 \operatorname{anos}^{(15)}$.

Quanto ao sexo, a presente pesquisa obteve resultados semelhantes a outros 


\begin{tabular}{|c|c|c|}
\hline Associação (NPH + Regular) & 2 & 1,9 \\
\hline Frequência de aplicações no diał & & $1,72 \pm 0,45$ \\
\hline \multicolumn{3}{|l|}{ Tipo de antidiabético } \\
\hline Metformina & 84 & 78,5 \\
\hline Glibencamida & 7 & 6,5 \\
\hline Glicazida & 2 & 1,9 \\
\hline Glimepirida & 1 & 0,9 \\
\hline Associações & 8 & 7,5 \\
\hline Frequência de comprimidos tomados no diat & & $2,05 \pm 0,57$ \\
\hline \multicolumn{3}{|l|}{$\begin{array}{l}\text { *Variáveis possiveis de mais de uma resposta. } \\
\text { 'Valores descritos em média } \pm \text { desvio-padrão. } \\
\text { Fonte: Dados da pesquisa }\end{array}$} \\
\hline \multicolumn{3}{|c|}{$\begin{array}{l}\text { Tabela } 4 \text { - Caracterização da amostra estudada, segundo as variáveis de tratament } \\
\text { Divinópolis - MG, } 2017 .\end{array}$} \\
\hline Variáveis & (N) & $(\%)$ \\
\hline \multicolumn{3}{|l|}{ Grupo de orientações de DM } \\
\hline Participa & 0 & 0 \\
\hline Já participou & 13 & 12,1 \\
\hline Nunca & 94 & 87,9 \\
\hline \multicolumn{3}{|l|}{ Problema para realizar o exame de glicemia } \\
\hline Sim & 7 & 6,5 \\
\hline Não & 37 & 34,6 \\
\hline Não possui aparelho & 63 & 58,9 \\
\hline \multicolumn{3}{|l|}{ Alterações nos pés } \\
\hline Nenhuma & 88 & 82,2 \\
\hline Rachadura & 15 & 13,9 \\
\hline Edema & 2 & 1,8 \\
\hline Descamação & 5 & 3,7 \\
\hline Ressecamento & 3 & 2,8 \\
\hline \multicolumn{3}{|l|}{ Amputações } \\
\hline Nenhuma & 105 & 98,1 \\
\hline Dedo MIE & 1 & 0,9 \\
\hline MID (abaixo joelho) & 1 & 0,9 \\
\hline Fonte: Dados da pesquisa. & & \\
\hline
\end{tabular}

Tabela 5 - Atividades de autocuidado de pacientes diabéticos acompanhados pelas Estratégias de Saúde da Família (ESF) da zona rural, Divinópolis - MG, 2017.

\section{Itens Avaliados}

\section{Alimentação geral}

Seguir dieta saudável

Seguir orientação nutricional de profissional

Alimentação específica

\begin{tabular}{ccc}
$\begin{array}{c}0-4 \text { dias } \\
\%\end{array}$ & $\begin{array}{c}5-7 \text { dias } \\
\%\end{array}$ & $\begin{array}{c}\text { Média de } \\
\text { dias/semana }\end{array}$ \\
\hline 36,4 & 63,6 & $4,74 \pm 2,25$ \\
\hline 46,7 & 53,2 & $4,33 \pm 2,32$ \\
\hline
\end{tabular}

estudos $^{(1,15)}$. Uma possível explicação seria pelo fato das mulheres preocuparem mais com a saúde, realizarem melhor o autocuidado e assim, buscarem constantemente pelos serviços de saúde e a assistência que the são oferecidas ${ }^{(1)}$. A prevalência das pessoas casadas pode estar ligada ao apoio familiar, que é um dos fatores importantes para o autocuidado. Estudos demonstram que as pessoas diabéticas que tiveram suporte adequado de seus familiares aderiam melhor às práticas do autocuidado e este tipo de conduta pode auxiliar na identificação das necessidades da assistência ${ }^{(16)}$.

Em relação ao ensino fundamental incompleto, segundo Cortez ${ }^{(17)}$, é válido destacar que devido à baixa escolaridade, os pacientes podem ter dificuldades para compreender as recomendações terapêuticas dadas pelos profissionais de saúde, o que justificaria a menor adesão desses pacientes ao tratamento.

Quanto à principal atividade exercida atualmente, do lar, ou seja, pessoas que se dedicam às atividades da casa, pode ser explicado pelo fato da maioria das pessoas pesquisadas já serem idosos e pelo diabetes mellitus ser uma doença que exige mudança de hábitos e complicações, gerando incapacidades e elevado custo terapêutico, podendo dificultar a capacidade de trabalho e a expectativa de vida, devido uma série de complicações ${ }^{(16)}$.

O número de pessoas residentes na mesma casa exerce papel determinante no cotidiano. Estudos ${ }^{(18)}$ destacam o papel importante da família no que tange ao seguimento das recomendações repassadas e auxilia na motivação para a adesão terapêutica, uma vez que o apoio e a participação da família repercutem positivamente para as melhorias das condutas do autocuidado. Da mesma forma que o número de habitantes tem implicação direta sobre o controle da doença no que se refere aos cuidados de higiene, alimentação e entre outras, uma vez que a renda seja inferior para atender a demanda familiar ${ }^{166)}$.

Diante dos resultados, em especial da baixa renda (0 a 3 salários mínimos) so- 


\begin{tabular}{|c|c|c|c|}
\hline 5 ou mais porções de frutas e/ou vegetais & 45,7 & 54,2 & $4,54 \pm 2,03$ \\
\hline $\begin{array}{l}\text { Alimentos ricos em gordura (carne vermelha, leite } \\
\text { integral e derivados) }\end{array}$ & 54,2 & 45,8 & $4,16 \pm 2,05$ \\
\hline Doces & 90,6 & 9,4 & $1,28 \pm 1,94$ \\
\hline \multicolumn{4}{|l|}{ Atividade física } \\
\hline $\begin{array}{l}\text { Pelo menos } 30 \text { minutos (total de atividade contínua, } \\
\text { inclusive andar) }\end{array}$ & 81,3 & 18,7 & $1,91 \pm 2,41$ \\
\hline Exercício físico espećíico (caminhar, andar de bicicleta, nadar) & 81,3 & 18,7 & $1,69 \pm 2,44$ \\
\hline \multicolumn{4}{|l|}{ Monitoramento da glicemia } \\
\hline Avaliar glicemia & 81,3 & 18,7 & $1,57 \pm 2,43$ \\
\hline Avaliar glicemia conforme recomendado & 80,5 & 19,6 & $1,60 \pm 2,50$ \\
\hline \multicolumn{4}{|l|}{ Cuidado com os pés } \\
\hline Examinar os pés & 40,2 & 59,9 & $4,45 \pm 3,07$ \\
\hline Examinar os sapatos antes de calçar & 34,6 & 65,5 & $4,89 \pm 2,98$ \\
\hline Secar espaços entre os dedos dos pés & 23,3 & 76,6 & $5,76 \pm 2,34$ \\
\hline \multicolumn{4}{|l|}{ Medicação } \\
\hline Tomou medicamentos/insulina, conforme recomendado & 3,7 & 96,3 & $6,66 \pm 1,25$ \\
\hline Tomou suas injeções de insulina, conforme recomendado & 1,8 & 21,6 & $6,60 \pm 1,13$ \\
\hline Ingerir quantidade indicada de comprimidos do diabetes & 2,8 & 92,5 & $6,79 \pm 0,70$ \\
\hline
\end{tabular}

Tabela 6 - Atividades de autocuidado referentes ao tabagismo de pacientes diabéticos acompanhados pelas Estratégias de Saúde da Família (ESF) da zona rural, Divinópolis - MG, 2017.

Itens Avaliados Tabagismo

$\%$

Fumou nos últimos 7 dias?

$\begin{array}{lc}\text { Não } & 92,5 \\ \text { Sim } & 7,5\end{array}$

\section{Quantidade de cigarros por dia}

\begin{tabular}{ll|}
\hline 1-2 cigarros & 2,8 \\
\hline 3-4 cigarros & 3,7 \\
\hline 5-6 cigarros & 2,8 \\
\hline Quando fumou o último cigarro & \\
\hline Nunca & 80,4 \\
\hline Há mais de dois anos & 7,5 \\
\hline Um a dois anos atrás & 2,8 \\
\hline Quatro a doze meses atrás & 0,9 \\
\hline No último mês & 0,9 \\
\hline Hoje & 7,5 \\
Fonte: Dados da pesquisa. &
\end{tabular}

mado o número de pessoas que habitam a casa, infere-se que se trata de pessoas que possuem um baixo índice de renda per capita. Segundo a OMS, tal quadro está estreitamente ligado a baixos níveis de escolaridade e precariedade da par- cela significante da população mundial. Indivíduos com baixo poder aquisitivo vivem e trabalham em ambientes de má-condições, em geral, não tem acesso aos serviços de saúde ou medidas preventivas. Mais, famílias carentes tendem a ter menor grau de aprendizagem e isso faz com que a saúde seja precária ${ }^{(18)}$.

No estudo, $68,2 \%$ dos diabéticos apresentaram valores de glicemia em jejum acima do que é estabelecido pela Associação Americana de Diabetes (ADA) para um adequado controle da patologia. E em relação à data da última glicemia em jejum, a maioria (44) relatou ter avaliado a glicemia há mais de um mês $(41,1 \%)$. Assim, quando níveis glicêmicos se encontram elevados, favorecerão o desencadeamento de complicações crônicas do DM, o que se faz importante o controle a partir de métodos que avaliam a frequência e a magnitude da hiperglicemia, sendo essenciais no acompanhamento da DM, assim como ajustes do tratamento(2,19).

Neste trabalho, em relação ao sobrepeso encontrado através da avaliação do IMC, segundo Braga ${ }^{(20)}$, a OMS mostra dados recentes de que o sobrepeso e a obesidade estão entre os cinco principais fatores de risco para o DM, com importante impacto na qualidade de vida. O estudo realizado por Fontela ${ }^{(21)}$ revelou que as pessoas diabéticas que possuem o IMC inadequado (sobrepeso/obesidade), apresentam duas vezes mais de chances de desenvolver complicações de diabetes do que as que possuem IMC adequado.

Quanto à circunferência abdominal, nas mulheres isto pode ser atribuído devido à maior concentração de gordura corporal, principalmente na região abdominal, devido às gestações e às diferenças hormonais, assim como o fato de que o processo de envelhecer ocasiona um declínio do hormônio do crescimento, da taxa metabólica basal e da redução natural dos níveis de atividade física, além dos hábitos saudáveis da alimentação, desta forma com a redistribuição progressiva da gordura, as mulheres passam a acumular mais gorduras abdominais ${ }^{(22)}$. 


\section{fisicamente ativos.}

De acordo com o tempo de diagnóstico e o tratamento, 76,6\% apresentavam a doença em menos de dez anos, estando em discordância com o estudo realizado por Cortez ${ }^{(17)}$ em que prevaleceram indivíduos com mais de 10 anos de diagnóstico da doença. Como tratamento a maioria $(95,3 \%)$ utilizava antidiabéticos orais, destes 78,5\% utilizavam a Metformina, o que condiz com o estudo realizado por Silva ${ }^{(24)}$ em que a metformina é apresentada como primeira escolha de medicamento para o tratamento do diabetes, pois apresenta características como discreta hiperglicemia, obesidade e insulinoresistência, além de reduzir $25 \%$ das complicações microvasculares.

Em relação aos problemas do tratamento, 63 (58,9\%) relataram como problema não possuir o aparelho, o que pode se atribuir ao fato do acesso aos insumos fornecidos pela saúde pública e também às dificuldades financeiras(25). No que tange às alterações nos pés, 88 (82,2\%) relataram que não tinham e 105 (98,1\%) não apresentavam amputações em nenhum dos membros. Isto pode ser considerado devido ao tempo da doença, pois estudos mostram que há maiores chances de desenvolvimento das lesões em pacientes com mais de 10 anos de acometimento da doença. Além de os pacientes apresentaram comportamento de autocuidado desejável (vide Tabela 4), pois as medidas adotadas na realização do autocuidado com os pés reduzem as taxas de desencadeamento das amputações ${ }^{(16)}$.

Em relação à monitorização da glice- mia, ao analisar os itens avaliar glicemia e avaliar glicemia conforme recomendado, observou baixa média de dias na semana, o que foi semelhante ao estudo realizado por Gomides ${ }^{(5)}$. Esse fato pode ser atribuído à maioria dos participantes ter relatado não possuir o aparelho em casa, como já discutido e apresentado na Tabela 4, assim como dificuldades para a realização da monitorização, no que diz respeito a fatores psicológicos, econômicos e sociais.

\section{6}

Ao considerar as atividades de autocuidado do uso da medicação, os itens avaliados obtiveram as maiores médias, verificando um comportamento de autocuidado desejável para o tratamento medicamentoso. índice de alterações nos pés e amputações discutido e relatado na Tabela 4, o que permite afirmar que os participantes sabem a importância do cuidado adequado com os pés para evitar futuras complicações decorrentes do DM. Sendo a prevenção a primeira linha contra úlceras diabéticas, podendo reduzir em até 50\% a ocorrência de lesões nos pés ${ }^{(16)}$.

Ao considerar as atividades de autocuidado do uso da medicação, os itens avaliados obtiveram as maiores médias, verificando um comportamento de autocuidado desejável para o tratamento medicamentoso. Assim, a pesquisa evidencia que as pessoas aderem mais ao tratamento medicamentoso do que a mudanças nos hábitos de vida (Tabela 5), pois muitas vezes esses estão arraigados desde a infância, além de representar uma mudança na rotina diária ${ }^{(4)}$.

Por fim, pode-se verificar durante a pesquisa considerações importantes sobre o perfil dos pacientes com DM e ainda sobre a adesão desses aos autocuidados de prevenção e tratamento à doença tais dados podem auxiliam na elaboração de estratégias para a diminuição dos sintomas, bem como para a maior e mais efetiva adesão dos pacientes, visando sempre à perspectiva de educação em saúde. Importa destacar ainda que, apesar de se verificar que muitos estavam informados quanto à necessidade de tratamento e prevenção do agravo da doença, considera-se que algumas estratégias poderiam ser melhor elaboradas e incentivadas pelos enfermeiros e profissionais da ESF, como o grupo de orientação do DM e ainda atividades de promoção à reflexão quanto aos autocuidados.

\section{CONCLUSÃO}

Os resultados do estudo permitiram

Quanto aos cuidados com os pés, todos os itens avaliados demonstraram média de dias maiores que 4, mostrando a realização de autocuidado desejável e entrando em concordância com o baixo verificar que a maioria dos pacientes com DM atendidos na EFSs na zona rural de Divinópolis são mulheres, idosas, com sobrepeso ou obesas, apresentam ainda HAS com diagnóstico de DM a menos 
de 5 anos. Em relação aos autocuidados, verificou-se que os pacientes aderem à estratégia medicamentosa, mas, embora não possuam comportamento de risco no que tange ao uso de bebidas alcóolicas e tabaco, também não mudaram de forma efetiva hábitos de vida que favorecem o agravo da doença como uma dieta gordurosa e inatividade.

Entretanto, nota-se a importância do profissional enfermeiro nos cuidados ao paciente com DM, sendo por meio de consulta de enfermagem e/ou assistência de enfermagem, o que irá proporcionar conhecimento da história pregressa e socioeconômica dos indivíduos para criar planos de cuidados específicos para cada paciente, estimulando a autonomia do paciente e capacitando os familiares para que os ajudem, principalmente com relação ao autocuidado e mudanças no hábito de vida das pessoas.

\section{Referências}

1. Ferreira DL, Rodrigues de Resende EAM, Lucas ALR, Silva ACF, Lenci SS, Silva SGF, Júnior FJ de A, Santos FHR dos, Passarelli FM, Bortolotto GM, Rezende I de P, Jardim J da C, Gonçalves RGLV, Messias LA. 0 efeito das equipes multiprofissionais em saúde no Brasil em atividades de cuidado com o diabetes. REAS [Internet]. 5dez.2018 [citado 19jul.2020];(17):e91. Disponível em: https://www.acervomais.com.br/index.php/saude/article/view/91 2. Sociedade Brasileira de Diabetes. DIRETRIZES DA SOCIEDADE BRASILEIRA DE DIABETES 2019-2020. 2019 [citado em 18 Jun 2020]. Disponível em: https://www.diabetes.org.br/profissionais/images/DIRETRIZES-COMPLETA-2019-2020.pdf

3. Ministério da Saúde (BR). Secretaria de Atenção à Saúde. Departamento de Atenção Básica. Diabetes Mellitus. Cadernos de Atenção Básica - n. 16. Série A. Normas e Manuais Técnicos. Brasília, DF: Ministério da Saúde; 2006 [citado em 18 nov 2019]. Disponível em: http://bvsms.saude.gov.br/bvs/publicacoes/diabetes_mellitus_cab16.pdf

4. Girardi CE, Heck R, Bobek ML, Benetti ERR, Stumm EMF, Colet CF. Qualidade de vida de pessoas em grupos de convivência com diabetes mellitus tipo 2. Rev Enferm UFPE on line. 2015;9(4):7239-46. Disponivel em: https://periodicos.ufpe.br/revistas/revistaenfermagem/article/viewFile/10484/11336 5. Gomides DS, Villas-Boas LCG, Coelho ACM, Pace AE. Autocuidado das pessoas com diabetes mellitus que possuem complicações em membros inferiores. Acta Paul Enferm. (Online). 2013;26(3):289-93. Disponível em: http:// www.scielo.br/pdf/ape/v26n3/14.pdf

6. Sousa JT, Macêdo SF, Moura JRA, Silva ARV, Vieira EES, Reis AS. Autocuidado e parâmetros clínicos em pacientes com diabetes mellitus tipo 2. Rev Rene (Online). 2015;16(4):479-85. Disponível em: http://www.periodicos. ufc.br/rene/article/view/2739/2122

7. Nascimento SR, Leite AHP, Silva NQ, Morato CBA, Rolim LADMM. Fatores de risco associados ao diabetes mellitus em adolescentes. Revista Brasilieira de Educação e Saúde. 2015;5(3):18-22. Disponível em: http://www.gvaa. com.br/revista/index.php/REBES/article/view/3645/3286

8. Oliveira GYM, Almeida AMO, Girão ALA, Freitas CHA. Intervenções de enfermagem para promoção do autocuidado de pessoas com diabetes tipo 2 : revisão integrativa. Rev Eletrônica Enferm. 2016;18:1188. Disponível em: https://revistas.ufg.br/fen/article/view/38691

9. Instituto Brasileiro De Geografia e Estatística. População recenseada e estimada, segundo os municípios 2017. Minas Gerais: IBGE; 2020 [citado em 18 jun 2020]. Disponível em: www.ibge.gov.br

10. Secretaria Municipal de Saúde de Divinópolis. Bases de dados Hiperdia 2017. Divinópolis: SMS; 2017.

11. Barbetta PA. Estatística aplicada às ciências sociais. 7a ed. Florianópolis: Editora UFSC, 2007.

12. Chibante CIP, Sabóia VM, Teixeira ER, Silva JLL. Qualidade de vida de pessoas com diabetes mellitus. Rev. Baiana Enferm. 2014;28(3):235-243. Disponível em: https://portalseer.ufba.br/index.php/enfermagem/article/ view/11909

13. Michels JM, Coral MHC, Sakae TM, Damas TB, Furlanetto LM. Questionário de atividades de autocuidado com o diabetes: tradução, adaptação e avaliação das propriedades psicrométricas. Arq Bras Endocrinol Metab. 2010;54(7):644-665. Disponível em: http://www.scielo.br/scielo.php?scrip- $\mathrm{t}=$ sci_arttext\&pid=S0004-27302010000700009

14. Parati G, Stergiou GS, Asmar R, Bilo G, Leeuw P, Imai Y, et al. European Society of Hypertension guidelines for blood pressure monitoring at home: a summary report of the Second International Consensus Conference on Home Blood Pressure Monitoring. J Hypertens. 2008;26:1505-1526. Disponível em:https://journals.Iww.com/jhypertension/fulltext/2008/08000/European_Society_of_Hypertension_guidelines_for.1.aspx

15. Loyola Filho Al, Firmo JOA, Lima-Costa MF. Fatores associados à autoavaliação negativa da saúde entre idosos hipertensos e/ou diabéticos: resultados do projeto Bambuí. Rev Bras Epidemiol. 2013;16(3): 559-71. Disponível em: http://www.scielo.br/scielo.php?pid=S1415=790-2013000300559X\&scriptsci_abstract\&tlng=pt

16. Santos ICRV, Sobreira CMM, Nunes ENS, Morais MCA. Prevalência e fatores associados a amputações por pé diabético. Ciênc Saúde Colet. 2013;18(10):3007-3014. Disponivel em: http://www.scielo.br/pdf/cscl v18n10/v18n10a25.pdf

17. Cortez DN, Reis IA, Souza DA, Macedo MM, Torres HC. Complicações e o tempo de diagnóstico do diabetes mellitus na atenção primária.

Acta Paul Enferm. 2015;28(3):250-5. Disponível em: http://www.scielo.br/ pdf/ape/v28n3/1982-0194-ape-28-03-0250.pdf

18. Neta DSR, Silva ARV, Silva GRF. Adesão das pessoas com diabetes mellitus ao autocuidado com os pés. Rev Bras Enferm 2015;68(1):111-6. Disponível em: http://www.scielo.br/scielo.php?pid=S0034=71672015000100111-\&scriptsci_abstract\&tlng=pt

19. American Diabetes Association. Classification and diagnosis of diabetes. Diabetes Care. 2015; 38(Suppl 1):S8- S16. Disponível em: http://care.diabetesjournals.org/content/38/Supplement_1/S8

20. Braga DC, Panka M, Cabral S, Bonamigo EL, Bortolini SM. Avaliação da função renal em pacientes com diabetes mellitus em um município rural do meio oeste de Santa Catarina. ACM Arq Catarin Med 2016;45(3): 84-92. Disponível em: http://www.acm.org.br/acm/seer/index.php/arquivos/article/ view/114

21. Fontela PC, Winkelmann ER, Ott JN, Uggeri, DP. Estimativa da taxa de filtração glomerular em com diabetes mellitus tipo 2. AMB Rev Assoc Med Bras 2014;60(6):531-537. Disponivel em: http://www.scielo.br/scielo. php?pid=S0104-42302014000600531\&script=sci_abstract\&tlng=pt 22. Barroso TA, Marins LB, Alves R, Gonçalves ACS, Barroso SG, Rocha GS. Associação entre a obesidade central e a incidência de doenças e fatores de risco cardiovascular. Int J Cardiovasc Sci. 2017;30(5):416-424. Disponível em: http://www.scielo.br/pdf/ijcs/v30n5/pt_2359-4802-ijcs-30-05-0416.pdf 23. Pinho CPS, Diniz AS, Arruda IKG, Fillho MB, Coelho PC, Sequeira LAS, et al. Prevalência e fatores associados à obesidade abdominal em indivíduos na faixa etária de 25 a 59 anos do Estado de Pernambuco, Brasil. Cad. Saúde Pública. 2013;29(2):313-324. Disponível em: http://www.scielo.br/scielo. php?script=sci_arttext\&pid=S0102-311X2013000200018

24. Silva KO, Messias GC, Souza EP, Jesus NN, Santos GS. Avaliação da compreensão dos pacientes portadores do diabetes mellitus tipo II quanto ao tratamento farmacológico e não farmacológico. Rev Saúde.com. 2015;11(4):382-396. Disponível em: http://www.uesb.br/revista/rsc/ojs/index.php/rsc/article/view/295 